\title{
ОПРЕДЕЛЕНИЕ ДОЛИ ПЛОДОВОЙ ДНК В ПЛАЗМЕ КРОВИ БЕРЕМЕННОЙ ЖЕНЩИНЫ С ПОМОЩЬЮ ВЫСОКОПРОИЗВОДИТЕЛЬНОГО СЕКВЕНИРОВАНИЯ НАБОРА ЧАСТОТНЫХ ОДНОНУКЛЕОТИДНЫХ ПОЛИМОРФИЗМОВ
}

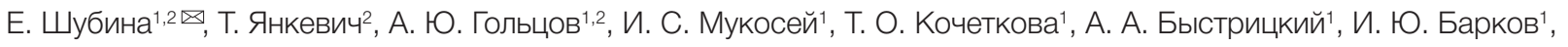 \\ Н. К. Тетруашвили ${ }^{1}$, Л. В. Ким¹, Д. Ю. Трофимов ${ }^{1,2}$ \\ 'Национальный медицинский исследовательский центр акушерства, гинекологии и перинатологии имени В. И. Кулакова, Москва \\ ${ }^{2} \mathrm{OОО} \mathrm{«НПФ} \mathrm{ДНК-Технология»,} \mathrm{Москва}$
}

\begin{abstract}
Неинвазивный пренатальный ДНК-скрининг (НИПС) анеуплоидий по крови матери применяется для выявления хромосомных анеуплоидий (ХА) с 2011 г. Многочисленные клинические исследования показали, что важным параметром при проведении НИПС является доля плодовой ДНК. Целью работы была разработка тест-системы для оценки доли плодовой ДНК с помощью таргетного секвенирования однонуклеотидных полиморфизмов (SNP). По данным исследований международного проекта НАРМАР были отобраны полиморфизмы с высокой частотой встречаемости гетерозиготного генотипа. Для оценки частоты встречаемости отобранных полиморфизмов в российской популяции использовали 827 образцов ДНК доноров. С целью определения доли плодовой ДНК исследовали 87 образцов плазмы крови беременных женщин. Секвенирование проводили на приборах lon Proton и lon S5. B ходе работы были определены частоты встречаемости по данным секвенирования пулированных образцов. Проведено сравнение данных о 53 SNP в 87 отдельных образцах. Медиана разницы, полученой различными способами, составила 3,4\%. Результаты определения доли плодовой ДНК с помощью SNP сравнивали с данными по Ү-хромосоме, корреляция составила 0,7. Таким образом, разработанную тест-систему можно применять для определения доли плодовой ДНК с помощью SNP вне зависимости от пола плода.
\end{abstract}

Ключевые слова: неинвазивный пренатальный ДНК-скрининг, доля плодовой ДНК, однонуклеотидные полиморфизмы, хромосомные анеуплоидии

Финансирование: работа поддержана Министерством образования и науки Российской Федерации (соглашение № 14.607.21.0136, идентификатор проекта RFMEFI60715X0136).

$\bowtie$ Для корреспонденции: Шубина Екатерина

ул. Академика Опарина, д. 4, г. Москва, 117997; jekaterina.shubina@gmail.com

Статья получена: 15.04.2018 Статья принята к печати: 18.04.2018

DOI: $10.24075 /$ vrgmu.2018.031

\section{QUANTIFICATION OF FETAL DNA IN THE PLASMA OF PREGNANT WOMEN USING NEXT GENERATION SEQUENCING OF FREQUENT SINGLE NUCLEOTIDE POLYMORPHISMS}

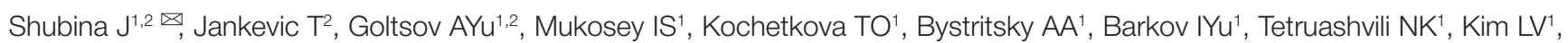
Trofimov DYu ${ }^{1,2}$

${ }^{1}$ Kulakov National Medical Research Center for Obstetrics, Gynecology and Perinatology, Moscow

${ }^{2}$ DNA-Technology LLC, Moscow

Introduced into clinical practice in 2011, non-invasive prenatal testing (NIPT) allows detection of chromosomal aneuploidies in the fetus using maternal blood samples. Multiple studies have shown that one of the key factors affecting the result of this test is the fetal DNA fraction. The aim of this work was to develop a method capable of measuring the fetal DNA fraction based on targeted SNP sequencing. We selected polymorphisms with high frequency of heterozygous genotype from the international HapMap database. To estimate the frequency of these polymorphisms in the Russian population, we used 827 DNA donor samples. Fetal DNA fraction was measured in 87 plasma samples of pregnant women. Sequencing was performed on lon Proton and Ion S5. We determined the frequencies of the studied polymorphisms in the pooled samples and compared the data on 53 SNPs in the pooled and 87 individual samples. The median difference was 3.4\%. The correlation between the results obtained by targeted SNP sequencing and $Y$ chromosome read count was 0.7 . Thus, the proposed method can be used to estimate the fetal DNA fraction using SNP genotyping regardless of the fetus's sex.

Keywords: non-invasive prenatal testing, fetal DNA fraction, single nucleotide polymorphisms, chromosome aneuploidy Funding: this work was supported by the Ministry of Education and Science of the Russian Federation (Agreement 14.607.21.0136, Project ID RFMEFI60715X0136).

$\checkmark$ Correspondence should be addressed: Jekaterina Shubina

Akademika Oparina 4, Moscow, 117997; jekaterina.shubina@gmail.com

Recieved: 15.04.2018 Accepted: 18.04.2018

DOI: $10.24075 /$ brsmu.2018.031 
Неинвазивный пренатальный ДНК-скрининг (НИПС) анеуплоидий по крови матери применяется для обнаружения хромосомных анеуплоидий (ХА) с 2011 г. [1]. Многочисленные клинические исследования показали, что важным параметром при проведении НИПС является доля плодовой ДНК [2, 3]. При недостаточном количестве плодовой ДНК снижается чувствительность теста и возможны ложноотрицательные результаты [3].

Долю плодовой ДНК просто определить в случае с беременностью плодом мужского пола. Для этого используют сравнение покрытия Ү-хромосомы с покрытием остальных аутосом. Если женщина вынашивает плод женского пола, то оценка представленности плодовых фрагментов становится более сложной задачей.

Известные способы определения представленности плодовой ДНК среди всей свободной циркулирующей ДНК (сцДНК) основаны на выявлении и подсчете фрагментов для которых можно определить их происхождение. Среди них использование фрагментов, специфичных для Y-хромосомы, которое возможно только для плода мужского пола; не зависящий от пола анализ по-разному метилированных фрагментов сцДНК [4], однонуклеотидных полиморфизмов [5-7], разницы длин фрггаментов плодовой и материнской ДНК [8], распределения фрагментов плодовой ДНК по геному [9-11].

С помощью таргетного секвенирования однонуклеотидных полиморфизмов (SNP) помимо определения доли плодовой ДНК становится возможной генетическая идентификация образца. Кроме того, этот же подход можно использовать для неинвазивного определения отцовства и пренатальной диагностики [12, 13].

Целью данной работы была разработка тест-системы, позволяющей определять долю плодовой ДНК вне зависимости от пола плода с помощью таргетного секвенирования SNP.

\section{МАТЕРИАЛЫ И МЕТОДЫ}

\section{Выбор однонуклеотидных полиморфизмов}

По данным широкомасштабных популяционных исследований HAPMAP [14] (http://hapmap.ncbi.nlm.nih.gov/) были отобраны 73 полиморфизма с частотой встречаемости гетерозиготного генотипа, равной 49-51\% для представителей популяции CEU (люди, произошедшие из западной и северной Европы), и 45-55\% для представителей африканской (ASW), китайской (CHD, CHB) и японской популяций (JPT); отобранные полиморфизмы находились на расстоянии не менее 20 млн п.н. на 1-12 хромосомах. Для каждого из отобранных полиморфизмов подобрали специфичные

Таблица 1. Результаты секвенирования пулированных образцов праймеры для амплисикации выбранного фррагмента. Праймеры отбирали таким образом, чтобы длина ПЦРпродукта была не более 110 п.н.

\section{Образцы ДНК и плазмы}

Оценку частоты встречаемости полиморфизмов в российской популяции проводили по результатам исследования 827 образцов ДНК, выделенных из крови доноров. Долю плодовой ДНК определяли в 87 образцах плазмы крови беременных женщин (в 45 образцах - у женщин с плодом мужского пола, в $42-$ у женщин с плодом женского пола).

\section{Оценка частоты встречаемости полиморфизмов в российской популяции}

Поскольку широкомасштабных популяционных данных о частоте встречаемости различных полиморфизмов в российской популяции нет и для отбора полиморфизмов использовали данные, полученные для других популяций, частота встречаемости выбранных полиморфизмов в исследуемой популяции может отличаться от опубликованных данных. В работе проводили оценку частоты встречаемости в российской популяции с помощью таргетного секвенирования смешанных (пулированных) в равных концентрациях образцов ДНК доноров.

Проводили секвенирование 10 пулов из 827 образцов (51-114 образцов/пул). Перед смешиванием ДНК оценивали ее концентрацию с помощью метода полимеразной цепной реакции (ПЦР) в реальном времени. Для оценки частоты встречаемости полиморфизмов в популяции суммировали частоты встречаемости, полученные для каждого пула, с учетом количества образцов в пуле. Результаты оценки частоты встречаемости полиморфизмов, полученные с помощью пулирования, сравнивали с результатами, полученными секвенированием 87 отдельных образцов.

\section{Определение доли плодовой ДНК}

Оценку доли плодовой ДНК проводили с помощью секвенирования 53 частотных полиморфизмов, которые были отобраны на основании предварительных данных секвенирования пулированных образцов.

Для определения доли плодовой ДНК использовали полиморфизмы, для которых представленность одного аллеля была более 80\%, но менее 99,5\%. В этом случае полагали, что мать имеет гомозиготный генотип, а плод гетерозиготный. Долю плодовой ДНК определяли по формуле $\mathrm{ff}=2 \cdot \mathrm{B} /(\mathrm{A}+\mathrm{B})$, где $\mathrm{A}$ - более представленный

\begin{tabular}{|c|c|c|c|}
\hline Номер пула & Количество образцов & Количество ридов & Количество ридов/полиморфизм тед (q1-q3) \\
\hline 1 & 96 & 2681517 & $12476(5195-40260)$ \\
\hline 2 & 114 & 2002697 & $13408(4724-34127)$ \\
\hline 3 & 96 & 2711707 & $17753(7810-48959)$ \\
\hline 4 & 84 & 3037177 & $20001(6742-44910)$ \\
\hline 5 & 78 & 3884900 & 28124 (10032-66108) \\
\hline 6 & 96 & 1677467 & $9808(2860-24624)$ \\
\hline 7 & 92 & 1592401 & $8826(2503-26345)$ \\
\hline 8 & 63 & 1759487 & $11359(3629-28146)$ \\
\hline 9 & 57 & 2340385 & $14355(3983-38147)$ \\
\hline 10 & 51 & 2403795 & $16195(4686-37680)$ \\
\hline
\end{tabular}


аллель, В - менее представленный аллель. В качестве значения доли плодовой ДНК использовали медиану значений для всех информативных полиморфизмов. Значение доли плодовой ДНК, определенные с помощью SNP, сравнивали со значением доли плодовой ДНК, определенным по покрытию Ү-хромосомы.

\section{Секвенирование}

Библиотеки из ПЦР-продуктов подготавливали согласно рекомендациям производителя (Thermo Fisher Scientific Inc., США). Секвенирование проводили на приборах Ion Proton и lon S5 (Thermo Fisher Scientific Inc., США) согласно протоколу производителя.

\section{Анализ данных}

Первичный анализ данных осуществляли с помощью программного обеспечения Torrent Server 4.4.3. Выравнивание на референсный геном версии GRCh37/hg19 проводили с помощью программного модуля ТМАР (Thermo Fisher Scientific Inc., США), после чего для позиций на геноме, соответствующих выбранным полиморфизмам, подсчитывали количество прочтений для каждого из аллелей (разработанный авторами скрипт с использованием программного пакета pysam [15]), для анализа отбирали фрагменты только с качеством выравнивания не менее 30 и с длиной не менее $80 \%$ от ожидаемой длины фрагмента.

\section{РЕЗУЛЬТАТЫ ИССЛЕДОВАНИЯ}

\section{Секвенирование пулированных образцов}

Результаты секвенирования пулированных образцов представлены в табл. 1.

После оценки работы системы в целом и частоты встречаемости отдельныхполиморфизмов для дальнейшей работы было отобрано 53 SNP. Исключены полиморфизмы с низкой представленностью. В табл. 2 представлены результаты сравнения частоты встречаемости для 53 SNP по данным секвенирования пулированных образцов и по данным анализа 87 отдельных образцов. Медиана разницы полученной двумя способами частоты встречаемости составила 3,4\%.

\section{Результаты определения доли плодовой ДНК}

Среднее количество полиморфизмов, для которых у матери был гомозиготный генотип, составило 28 (25-32), из них информативных полиморфизмов было 14 (10-18). На

Таблица 2. Сравнение частот встречаемости SNP по данным секвенирования пулированных образцов и по данным для 87 отдельных образцов

\begin{tabular}{|c|c|c|c|c|c|c|c|c|c|}
\hline \multirow{2}{*}{ номер SNP } & \multicolumn{2}{|c|}{ Пулированые образцы (827) } & \multicolumn{2}{|c|}{ отдельные образцы (87) } & \multirow{2}{*}{ номер SNP } & \multicolumn{2}{|c|}{ Пулированые образцы (827) } & \multicolumn{2}{|c|}{ отдельные образцы (87) } \\
\hline & аллель1 & аллель2 & аллель1 & аллель2 & & аллель1 & аллель2 & аллель1 & аллель2 \\
\hline rs4846002 & 0,619 & 0,381 & 0,592 & 0,408 & rs1265758 & 0,599 & 0,4 & 0,576 & 0,424 \\
\hline rs4926658 & 0,577 & 0,423 & 0,529 & 0,471 & rs2143829 & 0,574 & 0,426 & 0,616 & 0,384 \\
\hline rs9434166 & 0,576 & 0,424 & 0,598 & 0,402 & rs591356 & 0,438 & 0,562 & 0,453 & 0,547 \\
\hline rs10753750 & 0,564 & 0,436 & 0,586 & 0,414 & rs9373116 & 0,579 & 0,421 & 0,494 & 0,506 \\
\hline rs1973943 & 0,532 & 0,467 & 0,494 & 0,506 & rs7770051 & 0,479 & 0,521 & 0,407 & 0,593 \\
\hline rs7597744 & 0,49 & 0,51 & 0,494 & 0,506 & rs16 & 0,516 & 0,484 & 0,506 & 0,494 \\
\hline rs2121304 & 0,56 & 0,44 & 0,558 & 0,442 & rs12333726 & 0,564 & 0,435 & 0,552 & 0,448 \\
\hline rs1726025 & 0,517 & 0,483 & 0,517 & 0,483 & rs6958027 & 0,593 & 0,407 & 0,523 & 0,477 \\
\hline rs11164111 & 0,513 & 0,487 & 0,494 & 0,506 & rs314320 & 0,724 & 0,276 & 0,75 & 0,25 \\
\hline rs981841 & 0,49 & 0,509 & 0,558 & 0,442 & rs625218 & 0,597 & 0,403 & 0,618 & 0,382 \\
\hline rs1978346 & 0,653 & 0,347 & 0,698 & 0,302 & rs7005848 & 0,457 & 0,542 & 0,5 & 0,5 \\
\hline rs9843942 & 0,565 & 0,435 & 0,523 & 0,477 & rs952559 & 0,547 & 0,453 & 0,612 & 0,388 \\
\hline rs6777416 & 0,587 & 0,413 & 0,616 & 0,384 & rs827584 & 0,72 & 0,279 & 0,7 & 0,3 \\
\hline rs957303 & 0,61 & 0,39 & 0,593 & 0,407 & rs9987271 & 0,577 & 0,422 & 0,541 & 0,459 \\
\hline rs1553212 & 0,514 & 0,486 & 0,448 & 0,552 & rs6559467 & 0,583 & 0,417 & 0,612 & 0,388 \\
\hline rs751834 & 0,561 & 0,438 & 0,663 & 0,337 & rs4132699 & 0,667 & 0,332 & 0,647 & 0,353 \\
\hline rs6771838 & 0,645 & 0,354 & 0,622 & 0,378 & rs10980011 & 0,599 & 0,4 & 0,571 & 0,429 \\
\hline rs7696439 & 0,629 & 0,37 & 0,663 & 0,337 & rs2583839 & 0,603 & 0,397 & 0,565 & 0,435 \\
\hline rs4864809 & 0,452 & 0,548 & 0,517 & 0,483 & rs7904536 & 0,793 & 0,207 & 0,941 & 0,059 \\
\hline rs17002804 & 0,484 & 0,516 & 0,541 & 0,459 & rs4917915 & 0,531 & 0,468 & 0,453 & 0,547 \\
\hline rs978373 & 0,497 & 0,502 & 0,459 & 0,541 & rs845085 & 0,681 & 0,319 & 0,724 & 0,276 \\
\hline rs4621390 & 0,607 & 0,393 & 0,57 & 0,43 & rs4333997 & 0,522 & 0,477 & 0,488 & 0,512 \\
\hline rs7703985 & 0,491 & 0,509 & 0,442 & 0,558 & rs602991 & 0,696 & 0,304 & 0,747 & 0,253 \\
\hline rs2962799 & 0,542 & 0,456 & 0,541 & 0,459 & rs2289300 & 0,716 & 0,283 & 0,647 & 0,353 \\
\hline rs902987 & 0,52 & 0,478 & 0,512 & 0,488 & rs7973612 & 0,704 & 0,296 & 0,765 & 0,235 \\
\hline rs6859147 & 0,551 & 0,449 & 0,547 & 0,453 & rs7971962 & 0,606 & 0,39 & 0,606 & 0,394 \\
\hline rs4921132 & 0,495 & 0,505 & 0,494 & 0,506 & & & & & \\
\hline
\end{tabular}




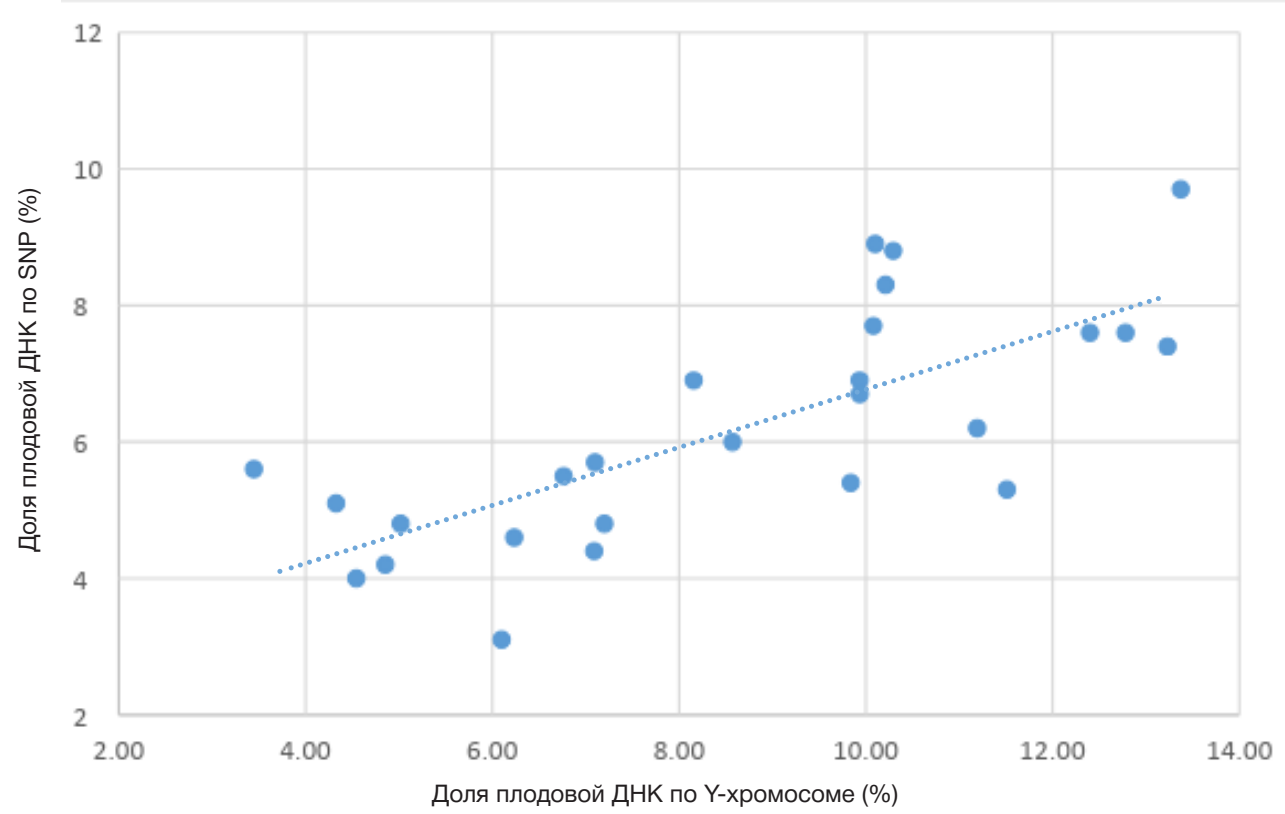

Рис. 1. Сравнение определения доли плодовой ДНК по Ү-хромосоме и с помощью таргетного секвенирования частотных полиморфизмов

рис. 1 представлено сравнение результатов определения доли плодовой ДНК с помощью SNP и по данным о покрытии Ү-хромосомы, корреляция составила 0,7.

\section{ОБСУЖДЕНИЕ РЕЗУЛЬТАТОВ}

Проведена оценка встречаемости выбранных полиморфизмов в исследуемой популяции с помощью секвенирования пулированных образцов. Продемонстрировано, что при пулировании и при определении генотипов отдельных образцов получаются сопоставимые результаты. Показана возможность оценки доли плодовой ДНК с помощью таргетного секвенирования небольшого количества частотных полиморфизмов вне зависимости от пола плода. Корреляция результатов определения доли плодовой ДНК с применением таргетного секвенирования и по
Ү-хромосоме в нашей работе ниже, чем по упоминающимся в литературе данным об использовании сопоставимого количества полиморфизмов для определения доли плодовой ДНК [7], что можно объяснить использованием в работе этих авторов молекулярных индексов и подсчета отдельных молекул.

\section{ВЫВОДЫ}

Предложенный метод можно использовать для оценки частоты встречаемости аллелей в случае частотных полиморсизмов. Помимо определения доли плодовой ДНК, описанный подход может быть применен для идентификации образцов, неинвазивного определения отцовства и неинвазивной диапностики наследственных заболеваний в случае наследования от отца мутации, которой нет у матери.

\section{Литература}

1. Agarwal A, Sayres LC, Cho MK, Cook-Deegan R, Chandrasekharan S. Commercial landscape of noninvasive prenatal testing in the United States. Prenat Diagn. 2013; 33 (6): 521-31.

2. Canick JA, Palomaki GE, Kloza EM, Lambert-Messerlian GM, Haddow JE. The impact of maternal plasma DNA fetal fraction on next generation sequencing tests for common fetal aneuploidies. Prenat Diagn. 2013; 33 (7): 667-74.

3. Сухих Г. Т., Каретникова Н. А., Баранова Е. Е., Шубина Е. С., Коростин Д. О., Екимов А. Н. и др. Неинвазивная пренатальная диагностика анеуплоидий методом высокопроизводительносго секвенирования (NGS) в группе женщин высокого риска. Акушерство и гинекология. 2015; 4: 5-10.

4. Nygren AO, Dean J, Jensen TJ, Kruse S, Kwong W, van den Boom D, et al. Quantification of fetal DNA by use of methylationbased DNA discrimination. Clin Chem. 2010 Oct; 56 (10): 1627-35.

5. Sparks AB, Struble CA, Wang ET, Song K, Oliphant A. Noninvasive prenatal detection and selective analysis of cell-free DNA obtained from maternal blood: evaluation for trisomy 21 and trisomy 18 . Am J Obstet Gynecol. 2012 Apr; 206 (4): 319. e1-9.

6. Nicolaides $\mathrm{KH}$, Syngelaki A, Gil M, Atanasova $\mathrm{V}$, Markova $\mathrm{D}$. Validation of targeted sequencing of single-nucleotide polymorphisms for non-invasive prenatal detection of aneuploidy of chromosomes 13, 18, 21, X, and Y. Prenat Diagn. 2013 Jun; 33 (6): 575-9.

7. Song $Y$, et al. Quantitation of fetal DNA fraction in maternal plasma using circulating single molecule amplification and re-sequencing technology (cSMART). Clin Chim Acta. 2016; 456: 151-6.

8. Yu SCY, et al. Size-based molecular diagnostics using plasma DNA for noninvasive prenatal testing. Proc Natl Acad Sci USA. 2014 Jun; 111 (23): 8583-8.

9. Kim SK, et al. Determination of Fetal DNA Fraction from the Plasma of Pregnant Women using Sequence Read Counts. Prenat Diagn. 2015: n/a-n/a.

10. Straver R, Oudejans CBM, Sistermans EA, Reinders MJT. Calculating the fetal fraction for Non Invasive Prenatal Testing based on Genome-wide nucleosome profiles. Prenat Diagn. 2016: n/a-n/a.

11. van Beek DM, et al. Comparing methods for fetal fraction determination and quality control of NIPT samples. Prenat Diagn. 2017; 37 (8): 769-73.

12. Lv W, et al. Noninvasive prenatal testing for Wilson disease by use of circulating single-molecule amplification and resequencing technology (cSMART). Clin Chem. 2015; 61 (1): 172-81. 
13. Meng $\mathrm{M}$, et al. Noninvasive prenatal testing for autosomal recessive conditions by maternal plasma sequencing in a case of congenital deafness. Genet Med. 2014; 16 (12): 972-6.

14. International HapMap Consortium. The International HapMap
Project. Nature. 2003; 426 (6968): 789-96.

15. Li H, et al. The Sequence Alignment/Map format and SAMtools. Bioinformatics. 2009 Aug; 25 (16): 2078-9.

\section{References}

1. Agarwal A, Sayres LC, Cho MK, Cook-Deegan R, Chandrasekharan S. Commercial landscape of noninvasive prenatal testing in the United States. Prenat Diagn. 2013; 33 (6): 521-31.

2. Canick JA, Palomaki GE, Kloza EM, Lambert-Messerlian GM, Haddow JE. The impact of maternal plasma DNA fetal fraction on next generation sequencing tests for common fetal aneuploidies. Prenat Diagn. 2013; 33 (7): 667-74.

3. Sukhikh GT, et al. Noninvasive prenatal diagnosis of aneuploidies by next-generation sequencing (NGS) in a group of high-risk women."Obstetrics and Gynecology. 2015; 4: 5-10.

4. Nygren AO, Dean J, Jensen TJ, Kruse S, Kwong W, van den Boom D, et al. Quantification of fetal DNA by use of methylationbased DNA discrimination. Clin Chem. 2010 Oct; 56 (10): 1627-35.

5. Sparks AB, Struble CA, Wang ET, Song K, Oliphant A. Noninvasive prenatal detection and selective analysis of cell-free DNA obtained from maternal blood: evaluation for trisomy 21 and trisomy 18 . Am J Obstet Gynecol. 2012 Apr; 206 (4): 319. e1-9.

6. Nicolaides $\mathrm{KH}$, Syngelaki A, Gil M, Atanasova V, Markova D. Validation of targeted sequencing of single-nucleotide polymorphisms for non-invasive prenatal detection of aneuploidy of chromosomes 13, 18, 21, X, and Y. Prenat Diagn. 2013 Jun; 33 (6): 575-9.

7. Song $Y$, et al. Quantitation of fetal DNA fraction in maternal plasma using circulating single molecule amplification and re-sequencing technology (cSMART). Clin Chim Acta. 2016; 456: 151-6.

8. Yu SCY, et al. Size-based molecular diagnostics using plasma DNA for noninvasive prenatal testing. Proc Natl Acad Sci USA. 2014 Jun; 111 (23): 8583-8

9. Kim SK, et al. Determination of Fetal DNA Fraction from the Plasma of Pregnant Women using Sequence Read Counts. Prenat Diagn. 2015: n/a-n/a.

10. Straver R, Oudejans CBM, Sistermans EA, Reinders MJT. Calculating the fetal fraction for Non Invasive Prenatal Testing based on Genome-wide nucleosome profiles. Prenat Diagn. 2016: n/a-n/a.

11. van Beek $D M$, et al. Comparing methods for fetal fraction determination and quality control of NIPT samples. Prenat Diagn. 2017; 37 (8): 769-73.

12. Lv W, et al. Noninvasive prenatal testing for Wilson disease by use of circulating single-molecule amplification and resequencing technology (cSMART). Clin Chem. 2015; 61 (1): 172-81.

13. Meng $\mathrm{M}$, et al. Noninvasive prenatal testing for autosomal recessive conditions by maternal plasma sequencing in a case of congenital deafness. Genet Med. 2014; 16 (12): 972-6.

14. International HapMap Consortium. The International HapMap Project. Nature. 2003; 426 (6968): 789-96.

15. Li H, et al. The Sequence Alignment/Map format and SAMtools. Bioinformatics. 2009 Aug; 25 (16): 2078-9. 